\title{
Vernodalol mediates antitumor effects in acute promyelocytic leukemia cells
}

\author{
WENJUN WU $^{1 *}$, XIAOYAN HAN $^{1 *}$, CAI WU $^{2}$, GUOQING WEI $^{1}$, GAOFENG ZHENG $^{1}$,

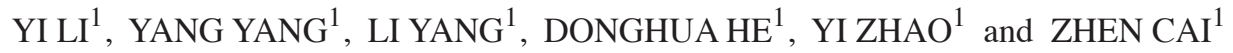

\begin{abstract}
${ }^{1}$ Department of Hematology, Bone Marrow Transplantation Center and Multiple Myeloma Treatment Center, The First Affiliated Hospital of Zhejiang Medical College, Zhejiang University, Hangzhou, Zhejiang 310003; ${ }^{2}$ Department of Hematology, The Fourth Affiliated Hospital of Zhejiang University School of Medicine, Yiwu, Zhejiang 322000, P.R. China
\end{abstract}

Received March 4, 2016; Accepted June 15, 2017

DOI: $10.3892 / 01.2017 .7544$

\begin{abstract}
Acute promyelocytic leukemia (APL) remains a challenge to cure due to the side effects of cytotoxic chemotherapy and drug resistance. The present study demonstrated that vernodalol, an active compound isolated from Centratherum anthelminticum, suppresses APL cell proliferation and induces cell cycle arrest in the G2/M phase through the upregulation of p21 and cell division cycle 25. In addition, vernodalol induced cellular apoptosis via the mitochondrial pathway as observed by the cleavage of caspase- 9 as well as the release of cytochrome $c$ and Smac/DIABLO into the cytosol. A mechanistic study revealed that vernodalol may exert its antitumor activity through the suppression of phosphoinositide 3-kinase/protein kinase $\mathrm{B} /$ mechanistic target of rapamycin signaling. In conclusion, vernodalol may be developed as a potential therapeutic compound for the treatment of APL.
\end{abstract}

\section{Introduction}

Acute promyelocytic leukemia (APL) is the M3 subtype of acute myeloid leukemia (AML) that results from an arrest of the terminal differentiation of promyelocytes into granulocytes $(1,2)$. APL accounts for $5-15 \%$ of all cases of AML and is frequently associated with the expression of the oncogenic promyelocytic leukemia-retinoic acid receptor $\alpha$ fusion gene $(3,4)$. Clinically, APL is now considered a fatal disease

Correspondence to: Professor Zhen Cai, Department of Hematology, Bone Marrow Transplantation Center and Multiple Myeloma Treatment Center, The First Affiliated Hospital of Zhejiang Medical College, Zhejiang University, 79 Qingchun Road, Hangzhou, Zhejiang 310003, P.R. China

E-mail: caiz@zju.edu.cn

*Contributed equally

Key words: acute promyelocytic leukemia, vernodalol, cell cycle, apoptosis, phosphoinositide 3-kinase/protein kinase B/mechanistic target of rapamycin with a high rate of early mortality, often due to hemorrhage from disseminated intravascular coagulation (DIC) or hyperfibrinolysis (5). Chemotherapy with anthracyclines, including daunorubicin, idarubicin and cytarabine arabinoside, which are the frontline treatments of APL, resulted in complete remission in $75-80 \%$ of patients $(6,7)$. However, this standard treatment regimen was associated with a high early mortality rate due to the exacerbation of pre-existing DIC $(5,7,8)$. Despite high sensitivity to anthracycline, only $35-45 \%$ of patients with APL are cured by standard chemotherapy alone (9). Therefore, it is urgently required to develop novel therapeutic agents for patients with APL to overcome the high early mortality rate.

Centratherum anthelminticum. commonly termed kalajiri, somraj, black cumin or bitter cumin, is a robust leafy plant belonging to the Asteraceae family of flowering plants (10). The plant has additional scientific synonyms, including Vernonia anthelmintica and Conyza anthelmintica. It is widely used as an herbal medicine against cough, fever and diarrhea in a number of regions, including India, the Himalaya mountains, Khasi Hills, Sri Lanka and Afghanistan (11). The extracts from the seeds of $C$. anthelminticum have been demonstrated to possess various pharmacological properties, including analgesic, antipyretic, anti-inflammatory, antiviral and anti-filarial effects (12-15). A previous study reported that the chloroform fraction, but not the hexane or methanol fractions, from C. anthelminticum seeds exhibited antioxidant properties of inhibiting tumor necrosis factor- $\alpha$-induced human cancer cell growth by interrupting the activation of nuclear factor- $\kappa \mathrm{B}$ activation (16). Vernodalin is an active compound isolated through bioassay-guided fractionation, which can induce apoptosis in human breast cancer cells via the caspase pathway (10). In addition, vernodalin can induce cell cycle arrest and apoptosis in breast cancer cells through forkhead box O3a (11). Vernodalol is similar to vernodalin, and both can be extracted from $C$. anthelminticum (10). However, the effects of vernodalol on carcinoma cells have not been studied.

The present study investigated the antitumor effects of vernodalol on APL cells. The effect of vernodalol on the APL cell cycle and apoptosis was assessed, and the associated molecular mechanisms were investigated. Results of the present study indicated that vernodalol may be utilized as a potent medicine for the treatment of APL. 


\section{Materials and methods}

Reagents and cell culture. Vernodalol was obtained from Sigma-Aldrich (Merck KGaA, Darmstadt, Germany). Antibodies specific for caspase-9 (cat. no. CST 9502), caspase-3 (cat. no. CST 9662), cleaved-poly (ADP ribose) polymerase (PARP; cat. no. CST 9542), B-cell lymphoma-2 (Bcl-2; cat. no. CST 2872), Bcl-2-associated X protein (Bax; cat. no. CST 2774), Bcl-2-associated death promoter (Bad; cat. no. CST 9292) and Bcl-2 homologous antagonist killer (Bak; cat. no. CST 3814) were obtained from Cell Signaling Technology, Inc. (Danvers, MA, USA). Antibodies specific for phosphatase and tensin homolog (PTEN; sc-7974), myeloid cell leukemia-1 (Mcl-1; sc-12756), Bcl-2-like protein 11 (Bim; sc-130511), cytochrome $c$ (sc-13561) and second mitochondria-derived activator of caspase/direct IAP-binding protein with low pI (Smac/DIABLO; sc-22766) were purchased from Santa Cruz Biotechnology, Inc. (Dallas, TX, USA). Antibodies specific for phosphorylated (p)-protein kinase B (Akt; ab38449), Akt (ab8805), phosphoinositide 3-kinase (PI3K; ab86714) and mechanistic target of rapamycin (mTOR; ab2732) were purchased from Abcam (Cambridge, MA, USA). Anti-GAPDH antibody was obtained from Sigma-Aldrich (Merck KGaA; G9545). The Annexin V/propidium iodide (PI) binding kit was purchased from BD Biosciences (San Jose, CA, USA). All other chemicals of analytical grade were obtained from Sigma-Aldrich (Merck KGaA). Human APL cell lines, KG-1a, NB-4 and HL-60, were obtained from Shanghai Centre of Cell Resource, Chinese Academy of Sciences (Shanghai, China). All cell lines were maintained at $37^{\circ} \mathrm{C}$ in RPMI-1640 (Gibco; Thermo Fisher Scientific, Inc.) supplemented with $10 \%$ heat-inactivated fetal bovine serum (Sigma-Aldrich; Merck KGaA) and $1 \%$ penicillin/streptomycin in a humidified atmosphere containing 5\% $\mathrm{CO}_{2}$. All cells were passaged every 3 days.

Cell viability assay. The effects of vernodalol on viability of human APL cells were analyzed by MTT assay (Sigma-Aldrich; Merck KGaA). Briefly, $5 \times 10^{3}$ cells per well were plated on 96-well plates and treated with vernodalol at various concentrations $(20,40,60,80$ and $100 \mu \mathrm{M})$ for the indicated times $(24,48$ or $72 \mathrm{~h})$. The RPMI-1640 with the compounds or $0.5 \%$ dimethyl sulfoxide (DMSO; as the control treatment) was then replaced with $180 \mu \mathrm{l}$ of fresh media with $20 \mu \mathrm{l}$ of MTT solution (MTT dissolved in PBS at $5 \mathrm{mg} / \mathrm{ml}$ ) per well and incubated at $37^{\circ} \mathrm{C}$ for $4 \mathrm{~h}$. The MTT-containing medium was discarded, and DMSO (150 $\mu \mathrm{l} /$ well) was added to dissolve the newly formed formazan crystals. The absorbance of each well was determined by a microplate reader (Synergy H4; BioTek China, Beijing, China) at a wavelength of $590 \mathrm{~nm}$.

Cell cycle analysis. The cells were cultured on 6-well plates at $37^{\circ} \mathrm{C}$ to reach $70-80 \%$ confluence with RPMI-1640 and then treated with vernodalol at various concentrations $(25,50$ or $100 \mu \mathrm{M})$ for the indicated time. The vernodalol-treated and control cells were harvested by centrifugation for $5 \mathrm{~min}$ at $377 \mathrm{x} \mathrm{g}$ and room temperature and fixed in $4 \mathrm{ml}$ ice-cold $75 \%$ ethanol at $4^{\circ} \mathrm{C}$ overnight. The cells were stained with $200 \mu \mathrm{l}$ PI $\left(50 \mu \mathrm{g} / \mathrm{ml}\right.$; Sigma-Aldrich; Merck KGaA) at $37^{\circ} \mathrm{C}$ for $10 \mathrm{~min}$ and incubated with $20 \mu \mathrm{l}$ RNase $(1 \mathrm{mg} / \mathrm{ml}$; Sigma-Aldrich;
Merck KGaA) for removal of RNA in a $37^{\circ} \mathrm{C}$ water bath for 15-20 min. The cells were then analyzed by flow cytometry (FACScan; BD Biosciences). The results are presented as mean values from three independent experiments.

Cell apoptosis analysis. The cells were cultured $\left(5 \times 10^{5}\right)$ in each well of 6-well plates to 70-80\% confluence with RPMI-1640 and then treated with vernodalol at various concentrations $(0,50,75$ or $100 \mu \mathrm{M})$ for the indicated time $(24 \mathrm{~h})$. The vernodalol-treated and control cells (treated with DMSO only) were harvested by centrifugation for $5 \mathrm{~min}$ at $377 \mathrm{x} \mathrm{g}$ and room temperature and washed twice with cold 1X PBS. The cells were then stained with $5 \mu 1$ Annexin V and PI (BD Biosciences) for 15 min in dark conditions at room temperature according to the manufacturer's protocol and then subjected to analysis by flow cytometry (FACS Calibur; BD Biosciences). Data were analyzed using Flowjo software version 10.0 (Tree Star, Inc., Ashland, OR, USA). Early apoptosis was evaluated based on the percentage of cells with Annexin $\mathrm{V}^{+} / \mathrm{PI}^{-}$staining, and late apoptosis was evaluated based on the percentage of cells with Annexin $\mathrm{V}^{+} / \mathrm{PI}^{+}$staining.

Preparation of subcellular fractions. To separate the cytosolic and mitochondrial fractions, the cells were washed with ice-cold PBS. The cells were then lysed using Cell Lysis and Mitochondria Intact buffer $(250 \mathrm{mM}$ sucrose, $80 \mathrm{mM} \mathrm{KCl}$ and $50 \mu \mathrm{g} / \mathrm{ml}$ digitonin in PBS) on ice for $5 \mathrm{~min}$. The cell suspension was centrifuged at $377 \mathrm{x} \mathrm{g}$ for $5 \mathrm{~min}$ at $4^{\circ} \mathrm{C}$. The supernatant was removed and stored at $-20^{\circ} \mathrm{C}$ as the cytosolic fraction.

Western blot analysis. The cells were treated with vernodalol at different concentrations $(0,50,75$ or $100 \mu \mathrm{M})$ for the indicated time $(24 \mathrm{~h})$. The cells were extracted and lysed with CHAPS lysis buffer (Beyotime Institute of Biotechnology, Beijing, China) for $30 \mathrm{~min}$ on ice and then centrifuged at $12,000 \mathrm{x} \mathrm{g}$ for $15 \mathrm{~min}$ at $4^{\circ} \mathrm{C}$. The total protein concentration was determined with bicinchoninic acid protein assay kit (Beyotime Institute of Biotechnology). Equal amounts (30 $\mu \mathrm{g}$ per lane) of protein samples were subjected to 8-12\% SDS-PAGE electrophoresis and transferred to polyvinylidene fluoride membranes (EMD Millipore, Billerica, MA, USA). Each membrane was blocked with $10 \%$ non-fat milk at room temperature for $2 \mathrm{~h}$ and incubated with primary antibodies (caspase- 9 , caspase-3, PARP, Bcl-2, Bax, Bcl-2, Bad, Bak, PTEN, Bim, cytochrome $c$, Smac/DIABLO, Akt, PI3K, mTOR and GAPDH) at $4^{\circ} \mathrm{C}$ overnight, followed by incubation with goat anti-mouse (cat. no. SZ200) or anti-rabbit (cat. no. SZ2004) secondary antibodies (1:1,000 dilution; Santa Cruz Biotechnology, Inc.) conjugated to horseradish peroxidase at room temperature for $2 \mathrm{~h}$. The relative protein expression levels were quantified using Image-Pro Plus software version 6.0 (Media Cybernetics, Inc., Rockville, MD, USA) and normalized to GAPDH.

Statistical analysis. Statistical analysis was performed using GraphPad Prism version 5.01 (GraphPad Software, Inc., La Jolla, CA, USA) and expressed as the mean \pm standard deviation. The values of half-maximal inhibitory concentration $\left(\mathrm{IC}_{50}\right)$ were fitted using a nonlinear regression model with a sigmoidal dose response. The values were evaluated 
by one-way analysis of variance followed by Tukey's multiple comparison, which was utilized to determine the significance of controls and treated groups. $\mathrm{P}<0.05$ was considered to indicate a statistically significant difference.

\section{Results}

Vernodalol inhibits the growth of human acute promyelocytic leukemia cells. The antitumor effects of vernodalol were initially tested using three human APL cell lines, NB4, KG-1a and HL-60. Cells were treated with different concentrations of vernodalol or vehicle (DMSO) for 24, 48 and $72 \mathrm{~h}$, and the cell viability was determined by MTT assay. As shown in Fig. 1 , the treatment of three APL cell lines with vernodalol resulted in a dose- and time-dependent attenuation of cell viability. $\mathrm{IC}_{50}$ values were calculated and are listed in Table I. The results showed that vernodalol inhibits the growth of APL cells in a dose- and time-dependent manner $(\mathrm{P}<0.01)$. Among these three APL cell lines, HL-60 was more sensitive to vernodalol compared with NB4 and KG-1a cell lines. Therefore, HL-60 was selected for subsequent experiments.

Vernodalol induces G2/M growth arrest. To examine whether vernodalol inhibited proliferation through the induction of cell cycle arrest, the effect of vernodalol on the cell cycle was assessed. HL-60 cells were treated with different concentrations of vernodalol for $24 \mathrm{~h}$, and the DNA-based cell cycle was analyzed by flow cytometry following PI staining. Vernodalol caused a marked accumulation of cells in the G2/M phase in a dose-dependent manner (Fig. 2A and B). Western blot analysis was performed to examine the effect of vernodalol on possible cell cycle-associated targets, including p21, cyclin B1 and Cdc25. As shown in Fig. 2C and D, vernodalol induced the upregulation of p21 and $\mathrm{Cdc} 25$ and the downregulation of cyclin B1 in a dose-dependent manner. These data indicated that vernodalol may induce G2/M cell cycle arrest via cell cycle regulatory molecules in APL cells.

Vernodalol induces apoptosis through the mitochondrial apoptosis pathway. Next, it was examined whether vernodalol had an effect on cell apoptosis. HL-60 cells were stained with Annexin V/PI subsequent to being treated with different concentrations of vernodalol for $24 \mathrm{~h}$ and subjected to flow cytometric analysis. As shown in Fig. 3A and B, the proportion of cells in early apoptosis (Annexin $\mathrm{V}^{+} / \mathrm{PI}^{-}$; right lower quadrant), as well as late apoptosis (Annexin $\mathrm{V}^{+} / \mathrm{PI}^{+}$; right upper quadrant), was increased with the different concentrations of vernodalol in a dose-dependent manner. These data indicated that vernodalol is able to induce cell apoptosis in human APL cells.

To elucidate the mechanism by which vernodalol induced cell apoptosis, apoptosis-associated proteins were examined following treatment with vernodalol. The results indicated that vernodalol caused cleavage of caspase- 9 and -3 in HL-60 cells (Fig. 3C and D). In addition, cleaved PARP was also detected, which is a marker of cells undergoing apoptosis (Fig. 3C and D). This suggested that the caspase cascade and PARP inactivation are involved in vernodalol-mediated apoptosis. As demonstrated in Fig. 4, vernodalol downregulated the expression of Bcl-2 and Mcl-1 but upregulated Bim, Bax and Bad
Table I. $\mathrm{IC}_{50}$ values of vernodalol on NB4, KG-1a and HL-60 cell lines.

\begin{tabular}{lrrr}
\hline & \multicolumn{3}{c}{$\mathrm{IC}_{50}, \mu \mathrm{M}$} \\
\cline { 2 - 4 } Cell lines & \multicolumn{1}{c}{$24 \mathrm{~h}$} & \multicolumn{3}{c}{$48 \mathrm{~h}$} & $72 \mathrm{~h}$ \\
\hline NB4 & $65.72 \pm 4.4$ & $36.6 \pm 4.5$ & $17.06 \pm 4.0$ \\
KG-1a & $76.4 \pm 1.5$ & $42.53 \pm 4.5$ & $23.78 \pm 2.9$ \\
HL-60 & $67.83 \pm 2.9$ & $38.36 \pm 3.4$ & $16.43 \pm 3.5$ \\
\hline
\end{tabular}

$\mathrm{IC}_{50}$, half-maximal inhibitory concentration.
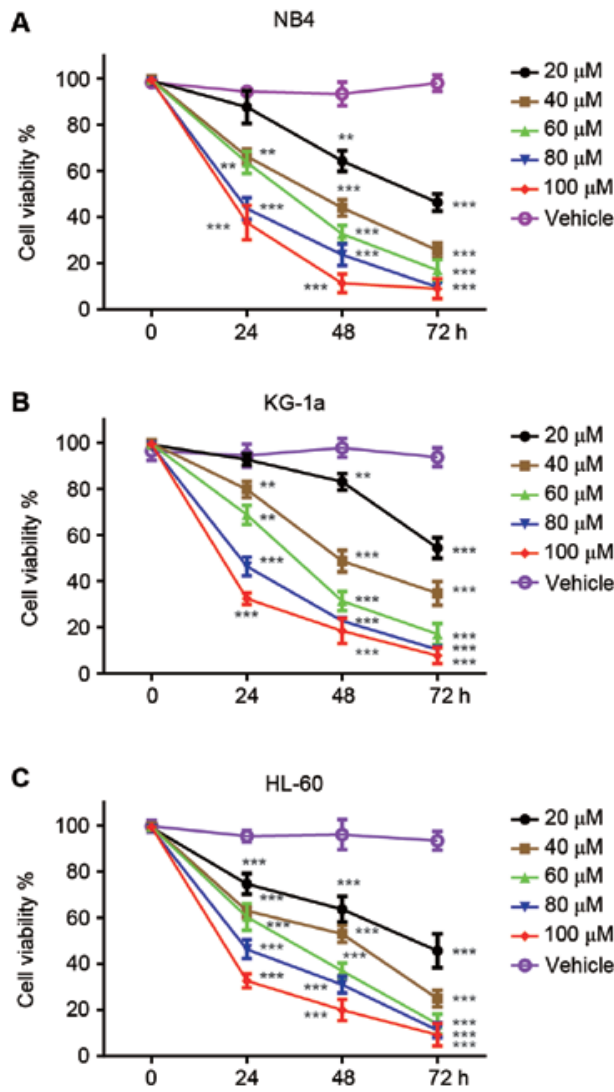

Figure 1. Anti-proliferative effects of vernodalol on three human acute promyelocytic leukemia cell lines. Human acute promyelocytic leukemia (A) NB4, (B) KG-1a and (C) HL-60 cells were treated with various concentrations of vernodalol $(20,40,60,80$ and $100 \mu \mathrm{M})$ for 24,48 and $72 \mathrm{~h}$. Viability was determined by MTT assay. Values are presented as the mean \pm standard deviation $(\mathrm{n}=3) .{ }^{* *} \mathrm{P}<0.01$ and ${ }^{* * *} \mathrm{P}<0.001$ vs. vehicle control.

expression in a dose-dependent manner. In addition, it was investigated whether the mitochondrial apoptosis pathway was involved in vernodalol-induced apoptosis. The release of cytochrome $c$ and Smac into the cytosol was measured following cell treatment with vernodalol. Vernodalol caused a significant increase in the expression of cytochrome $c$ and Smac/DIABLO in the cytosol (Fig. 4B and C). These results indicated that vernodalol induces cell apoptosis through the mitochondrial apoptosis pathway in APL cells.

Vernodalol inhibits the PI3K/Akt signaling pathway. It has been reported that the inhibition of the PI3K/Akt/mTOR signaling 
A

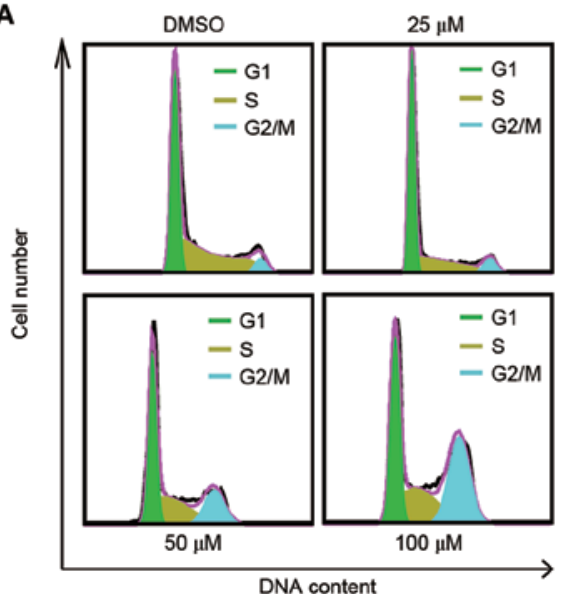

B

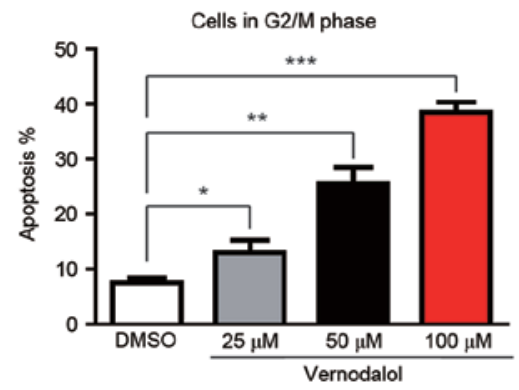

C

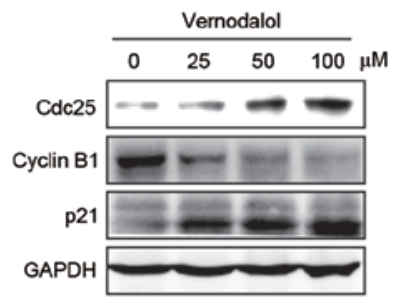

D

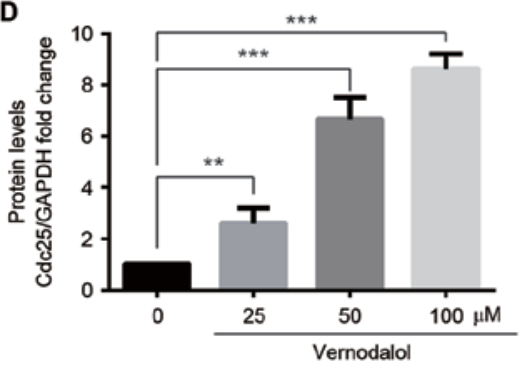

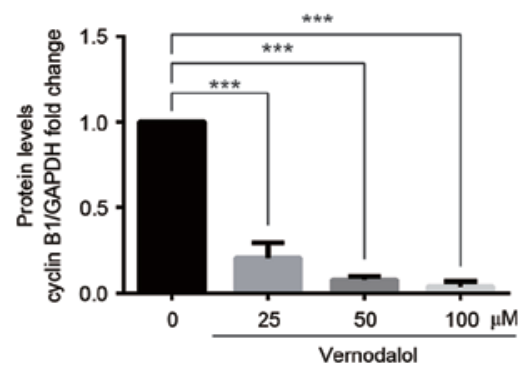

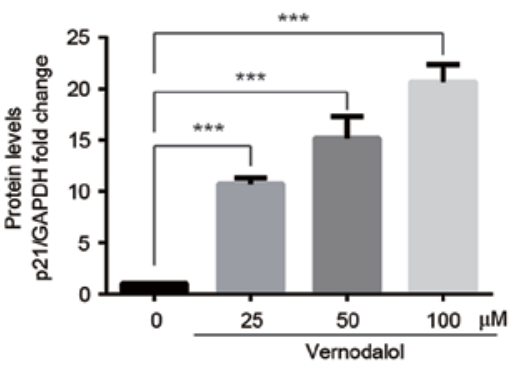

Figure 2. Vernodalol suppresses cell cycle progression in HL-60 cells. (A) Representative flow cytometry profiles of cell cycle distribution following treatment with 25,50 or $100 \mu \mathrm{M}$ vernodalol for $24 \mathrm{~h}$. (B) Quantitative analysis of the $\mathrm{G} 2 / \mathrm{M}$ phase following treatment with various concentrations of vernodalol (25, 50 and $100 \mu \mathrm{M}$ ) for $24 \mathrm{~h}$. (C) HL-60 cells were treated with various doses of vernodalol (25, 50 and $100 \mu \mathrm{M})$ for $24 \mathrm{~h}$. Cellular lysates were subjected to western blot analysis with the indicated antibodies. GAPDH was used as a loading control. (D) Quantitative analysis of Cdc25, cyclin B1 and p21. Protein expression was normalized to GAPDH using a densitometer. Data are presented as the mean \pm standard deviation $(\mathrm{n}=3) .{ }^{*} \mathrm{P}<0.05,{ }^{* * *} \mathrm{P}<0.01,{ }^{* * * *} \mathrm{P}<0.001$ vs. vehicle control. Cdc25, cell division cycle 25; DMSO, dimethyl sulfoxide.

pathway has a vital role in the inhibition of tumors (17). Therefore, western blot analysis was performed to investigate whether the PI3K/Akt/mTOR signaling pathway was involved in the vernodalol-mediated anticancer effects. The results demonstrated that the phosphorylation of Akt at Ser473 as well as the expression of mTOR, a well-known downstream target of Akt, were downregulated in cells treated with vernodalol in a dose-dependent manner (Fig. 5). Furthermore, there were little changes in the levels of PI3K-p110 $\alpha$ and total Akt. Additionally, PTEN, a key negative regulator of the PI3K/Akt pathway, was upregulated in the treated cells (Fig. 5) compared with the untreated cells. These results provide compelling preliminary data that vernodalol may exert its antitumor function through inhibition of the PI3K/Akt/mTOR signal pathway.

\section{Discussion}

APL is a distinct subtype of acute leukemia (9). Patients with APL exhibit a tendency to bleed severely and prognosis is poor with a fatal course in only weeks (5). Although the clinical outcomes of refractory, relapsed and newly diagnosed patients with APL have been greatly improved, the mortality rate remains high (18). The present study demonstrated that vernodalol isolated from C.anthelminticum exerted antitumor effects in human APL cells. Vernodalol suppressed growth of human APL cells by inducing apoptosis through the mitochondrial pathway and causing cell cycle arrest in the G2/M phase. In addition, the results suggested that the strong cytokinetic effects of vernodalol were in part mediated via inhibition of the PI3K/Akt signaling pathway.

Vernodalol is a sesquiterpene lactone isolated from the seeds of $C$. anthelmintica (19). To date, a limited number of studies, which investigate the anti-cancer effects of vernodalol in human breast cancer cells and skin cancer models have been performed $(10,20)$. These studies demonstrated the cytotoxic activity of vernodalol on melanoma and ovarian cancer cell lines, and human nasopharyngeal carcinoma $(21,22)$. However, the effect and the precise mechanism of vernodalol on human APL cells, remains unclear. To the best of our knowledge, this is the first study investigating the function and mechanism of vernodalol in human APL cells.

Cell cycle dysregulation contributes to tumor initiation and progression. Therefore, cell cycle arrest has become the major focus of anticancer treatments (23). The majority of chemotherapeutic agents cause cell cycle arrest either at the G0/G1 or G2/M stage $(24,25)$. In the present study, downregulation of cyclin B1 expression and upregulation of $\mathrm{p} 21$ and $\mathrm{Cdc} 25$, was observed following vernodalol treatment. p21, which belongs to the Cip/Kip family of cyclin-dependent kinase (CDK) inhibitors, binds to and inhibits the kinase activity of CDK2 and CDK1, leading to growth arrest at specific stages of the cell cycle (26). Cdc25C triggers G2 progression to mitosis by dephosphorylation of the cyclin B1/Cdk1 complex (26). Cyclin B1 is also a key cell cycle regulator of the G2/M phase transition, and the prototypical cyclin $\mathrm{B} 1$ reaches maximum levels in G2 when it enters the nucleus to form a complex 
A

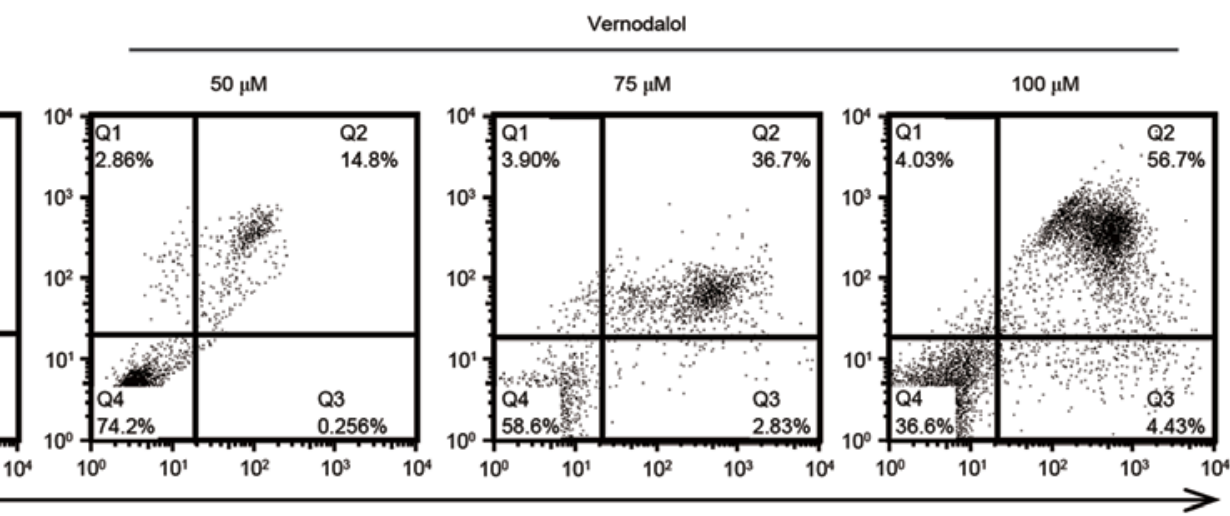

PI

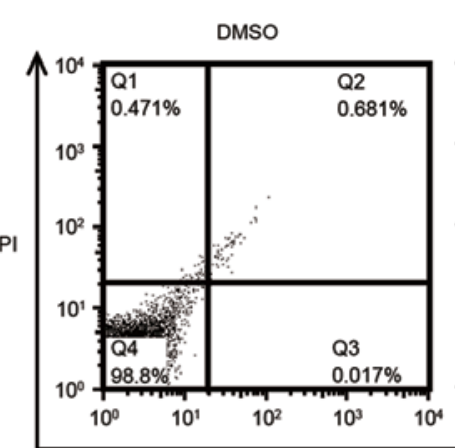

Annexin V-FITC

B

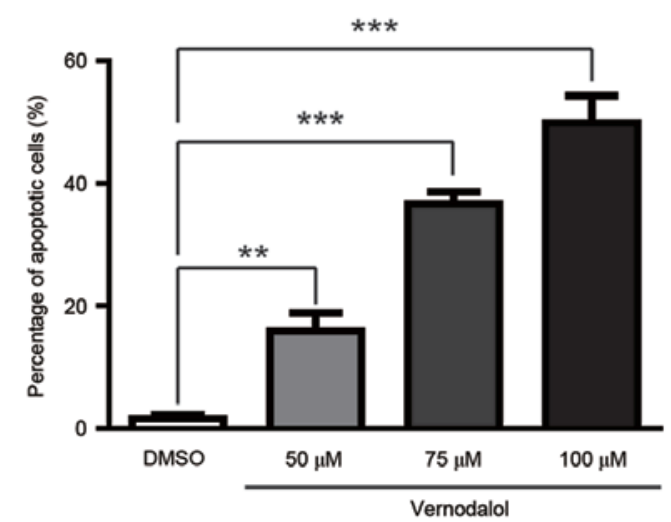

D
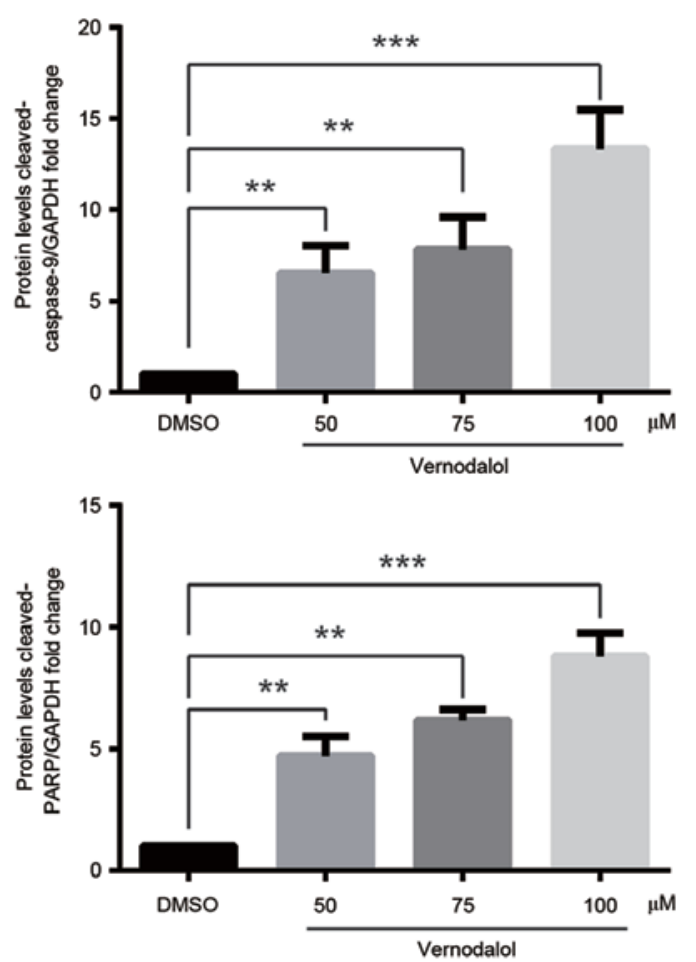

C
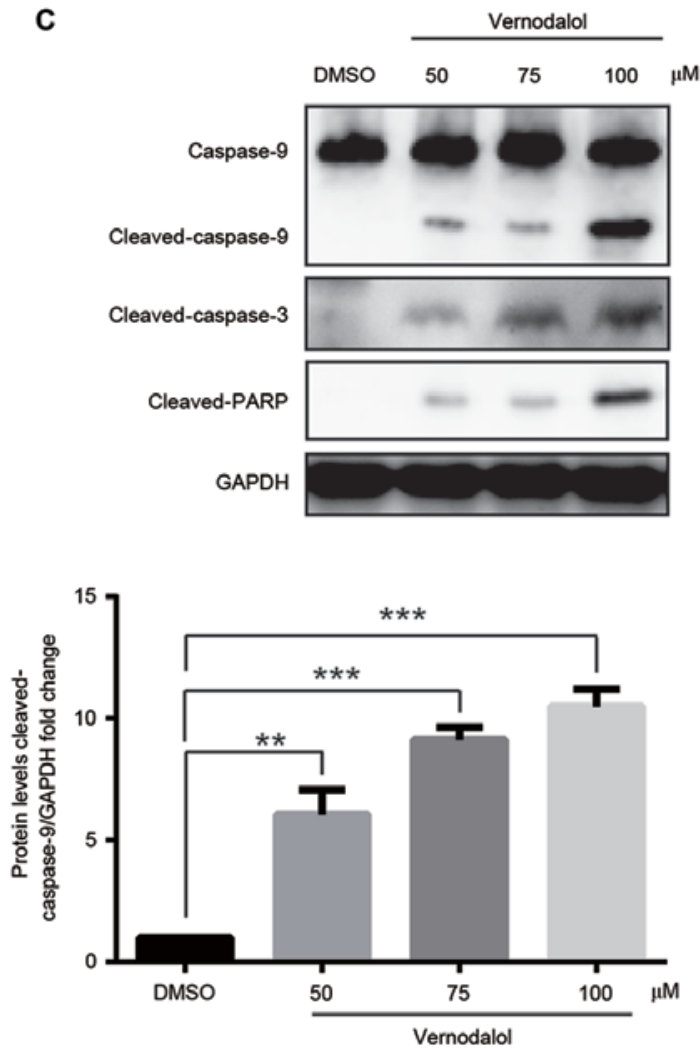

Figure 3. Vernodalol induces apoptosis in HL-60 cells. (A) Representative flow cytometry profiles of apoptosis in HL-60 cells induced by different concentrations of vernodalol for $24 \mathrm{~h}$. (B) Quantitative results were obtained using Annexin V/PI staining. (C) Following treatment with different concentrations of vernodalol for $24 \mathrm{~h}$, whole cellular extracts were subjected to western blot analysis with the indicated antibodies. GAPDH was used as a loading control. (D) Quantification analysis of cleaved-caspase-9, cleaved-caspase-3 and cleaved-PARP protein expression was normalized to GAPDH using a densitometer. Data are presented as the mean \pm standard deviation $(n=3) .{ }^{* *} \mathrm{P}<0.01,{ }^{* * *} \mathrm{P}<0.001$ vs. vehicle control. PI, propidium iodide; FITC, fluorescein isothiocyanate; DMSO, dimethyl sulfoxide; PARP, poly ADP ribose polymerase.

with Cdk1 in a phosphorylation-dependent manner (27). It has been reported that the overexpression of cyclin B1 causes uncontrolled cell growth and may even promote the malignant transformation required for the initiation of mitosis (28). Taken 
A

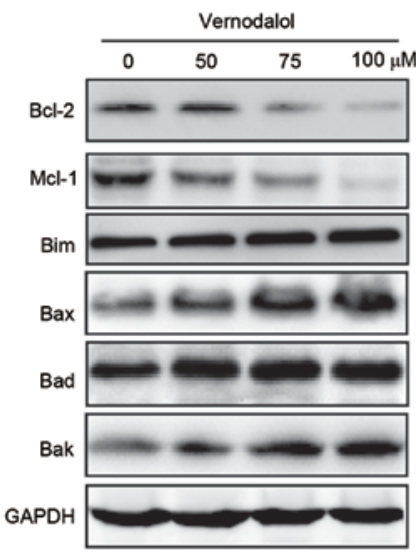

C
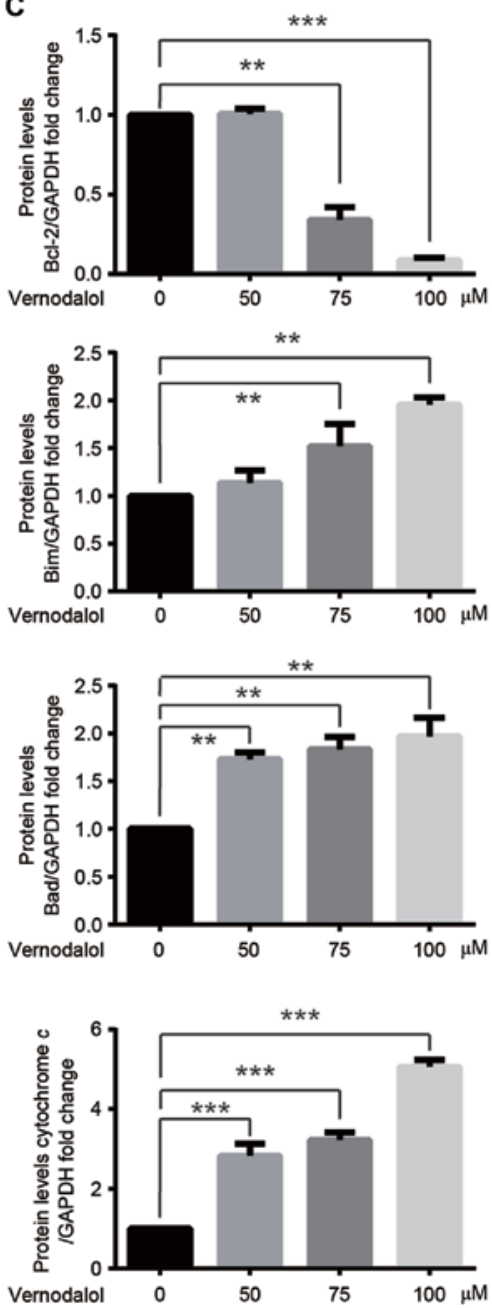

B

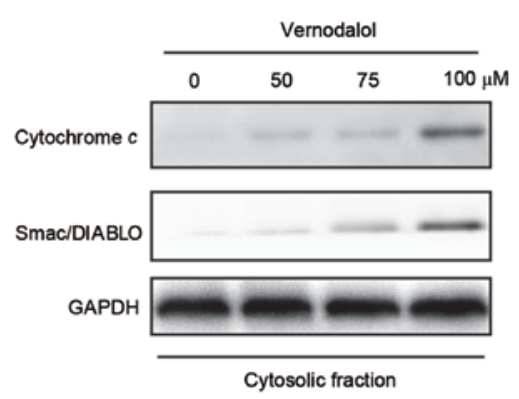

Cytosolic fraction
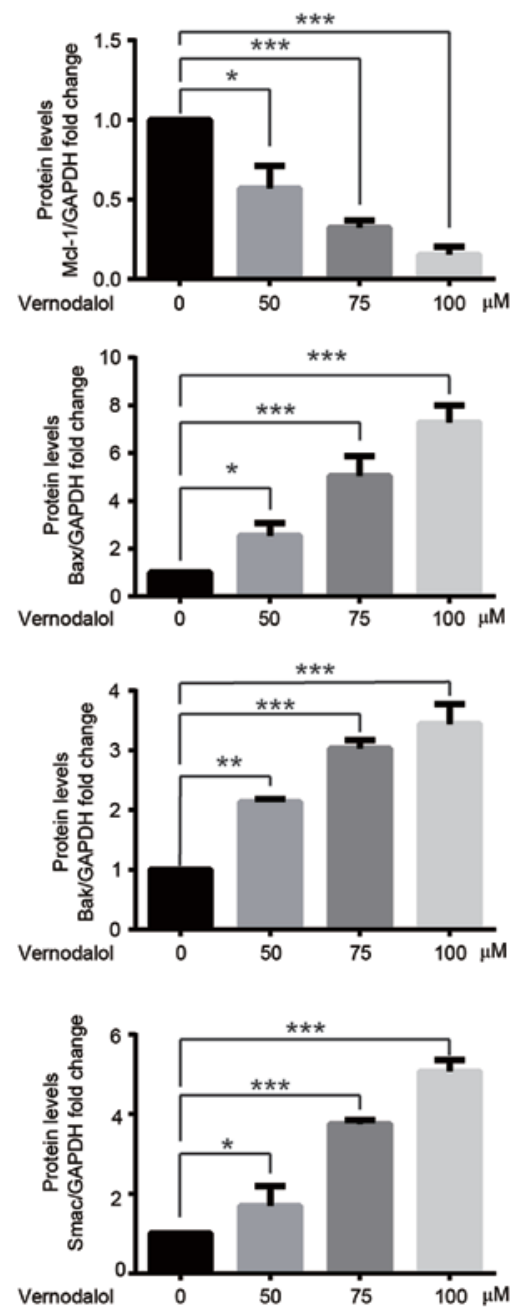

Figure 4. Vernodalol affects the expression of apoptosis-associated proteins. (A) HL-60 cells were treated with various concentrations of vernodalol. Cellular extracts were analyzed by western blot analysis with the indicated antibodies. (B) Cytosolic fractions were analyzed by western blot analysis with the indicated antibodies. (C) Quantification of Bcl-2, Mcl-1, Bim, Bax, Bad, cytochrome c and Smac/DIABLO protein expression was normalized to GAPDH using a densitometer. Data are presented as the mean \pm standard deviation $(n=3) .{ }^{*} \mathrm{P}<0.05,{ }^{* *} \mathrm{P}<0.01$ and ${ }^{* * *} \mathrm{P}<0.001$ vs. vehicle control. Bcl-2, B-cell lymphoma-2; Bax, Bcl-2-associated X protein; Bad, Bcl-2-associated death promoter; Mcl-1, myeloid cell leukemia-1; Bim, Bcl-2-like protein 11; Smac/DIABLO, second mitochondria-derived activator of caspase/direct IAP-binding protein with low pl.

together, the present data suggest a novel cell cycle regulating property of vernodalol via G2-phase arrest in human APL cells.

Apoptosis, a complex program of cell death, is regulated by numerous molecular signaling pathways (29). A study has shown that the mitochondrial pathway performs a key role in the apoptotic process (10). In this pathway, there is an increase in the release of apoptogenic factors (cytochrome $c$ and Smac/DIABLO) from the outer mitochondrial membrane space into the cytosol due to changes in the mitochondrial membrane potential (Dym). The Bcl-2 family is a major regulator of programmed cell death (30). A previous study reported that $\mathrm{Bcl}-2$ can block cell death in mitochondria by inhibiting the apoptosis-associated release of cytochrome $c$ from the mitochondria (30). Results of the present study indicated a 
A

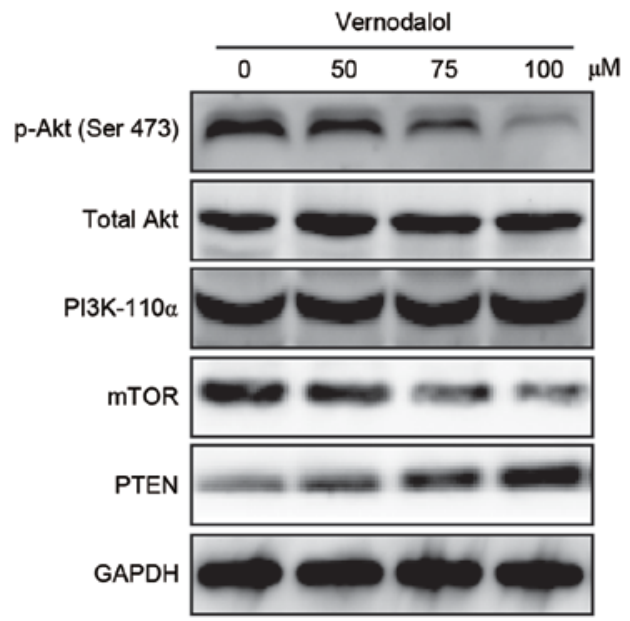

B
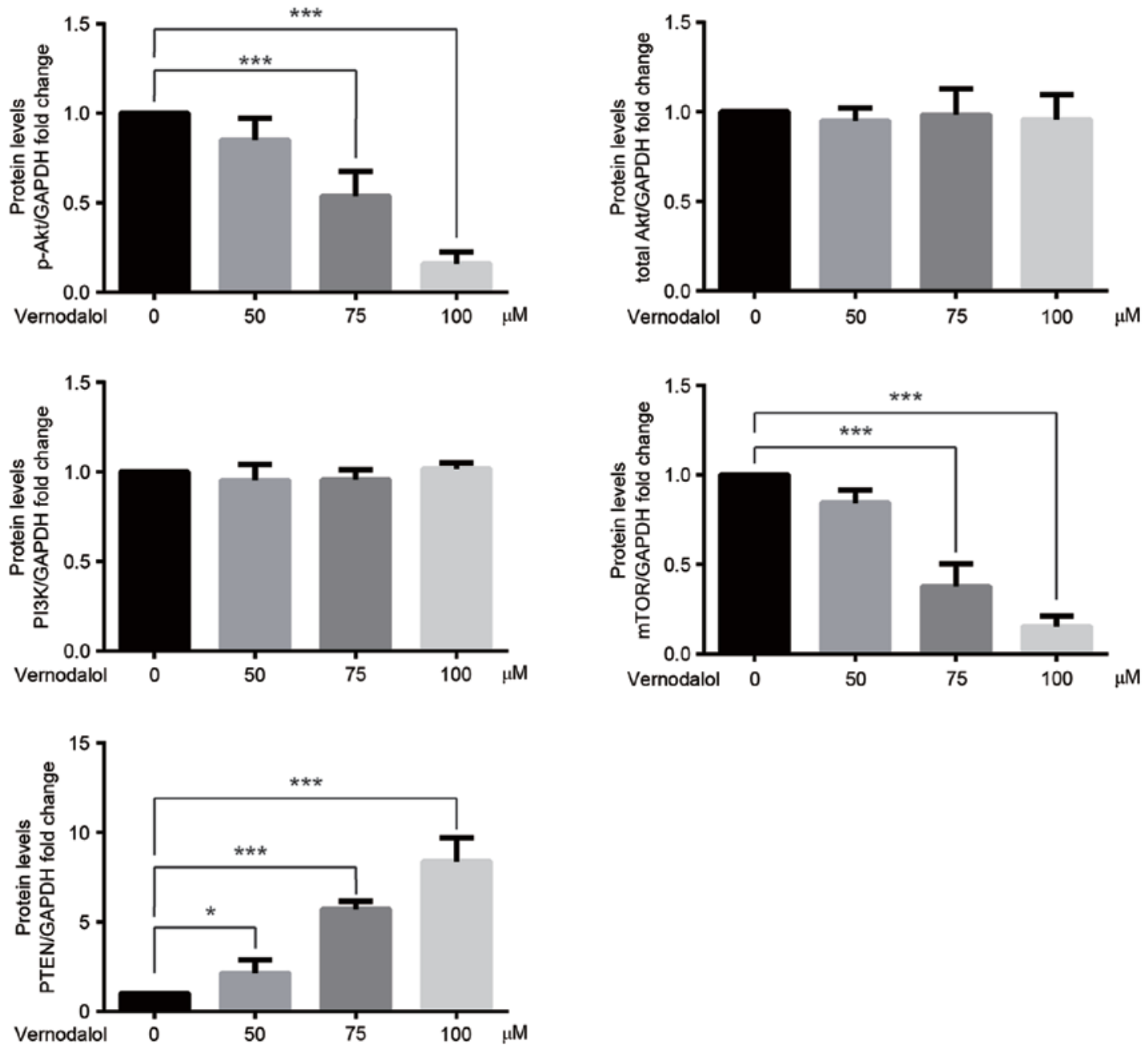

Figure 5. Vernodalol inhibits the PI3K/Akt/mTOR signaling pathway. (A) HL-60 cells were treated with various concentrations of vernodalol. Cellular extracts were analyzed by western blot analysis with the indicated antibodies. (B) Quantification of p-Akt, Akt, PI3K, mTOR and PTEN protein expression was normalized to GAPDH using a densitometer. Data are presented as the mean \pm standard deviation $(\mathrm{n}=3)$. ${ }^{*} \mathrm{P}<0.05,{ }^{* * * *} \mathrm{P}<0.001$ vs. vehicle control. PI3K, phosphoinositide 3-kinase; mTOR, mechanistic target of rapamycin; PTEN, phosphatase and tensin homolog; Akt, protein kinase B; p-Akt, phosphorylated-Akt.

decrease in the levels of anti-apoptotic proteins Bcl-2 and Mcl-1 and an increase in pro-apoptotic proteins Bim, Bax and Bad following treatment with vernodalol (Fig. 4A). Furthermore, the levels of cytochrome $c$ and Smac/DIABLO were increased in the cytosol following treatment with vernodalol. Therefore, the present findings indicated that treatment with vernodalol induces apoptosis in a manner that is dependent on activation of the mitochondrial apoptosis pathway.

The PI3K/Akt signaling pathway is a cell survival pathway that is important for normal cell growth and proliferation (31). Numerous studies have demonstrated that the PI3K/Akt signaling pathway is involved in various cellular functions, 
including protein synthesis, cell cycle progression, cell survival, apoptosis and angiogenesis $(31,32)$. The amplification of PI3K/Akt signal transduction is the main cause of cellular growth, and the aberrant activation of PI3K/Akt due to genetic variation in key genes has been observed in several types of cancer, including leukemia and esophageal cancer $(33,34)$. It has been reported that the PI3K/Akt signaling axis is constitutively activated in APL, and the addition of PI3K and mTOR inhibitors to induction treatment regimens may provide therapeutic benefits for APL $(33,35)$. Akt is one of the major downstream effectors of PI3K (36). Upon PI3K activation, Akt is translocated to the inner membrane via its pleckstrin homology domain where it is phosphorylated by 3-phosphoinositide-dependent kinase-1 on its activation loop (37). This Akt modification is sufficient to activate the mTOR complex 1 , which was originally identified as a crucial component of insulin receptor intracellular signaling (38). PTEN, a tumor suppressor phosphatase and tensin homolog, may antagonize PI3K/Akt signaling (39). PTEN was identified as a frequently mutated gene in numerous types of tumors, particularly endometrium, skin, brain and prostate (40). A study has also revealed that the downregulation of PI3K, Akt and mTOR and increased PTEN gene expression induces apoptosis and inhibits cell cycle progression in the HL-60 cell line (33). Therefore, the abrogation of PI3K or Akt function may be crucial for APL therapy. In the present study, western blot analysis demonstrated that vernodalol attenuated the phosphorylation of Akt at Ser473 and the expression of the downstream target mTOR and increased the expression of tumor suppressor PTEN (Fig. 5A) in a dose-dependent manner in human APL cells. These results suggest that vernodalol may induce apoptosis and inhibit cell cycle G2/M arrest by suppressing the PI3K/Akt signaling pathway. However, further investigation is required to validate this hypothesis.

In conclusion, to the best of our knowledge, the present study reported for the first time that vernodalol, an active compound isolated from $C$. anthelminticum, has a potential therapeutic function in human APL cells. Vernodalol induces cell apoptosis and G2/M growth arrest. In addition, the present findings also highlighted the 'therapeutic value of the repression of the PI3K/Akt pathway by vernodalol in human APL cells. Therefore, vernodalol may be a potential candidate used for the treatment of APL, subsequent to further investigation.

\section{Acknowledgements}

The present study was supported by the National Natural Science Foundation of China (grant no. 30900533), the Science Foundation of Traditional Medicine Zhejiang Province (grant no. 2013ZB080) and the Science Foundation of National Health and Family Planning Commission (grant no. 2014BAI09B12).

\section{References}

1. Bennett JM, Catovsky D, Daniel MT, Flandrin G, Galton DA, Gralnick HR and Sultan C: Proposals for the classification of the acute leukaemias. French-American-British (FAB) co-operative group. Br J Haematol 33: 451-458, 1976.

2. Vardiman JW, Thiele J, Arber DA, Brunning RD, Borowitz MJ, Porwit A, Harris NL, Le Beau MM, Hellström-Lindberg E, Tefferi A and Bloomfield CD: The 2008 revision of the World Health Organization (WHO) classification of myeloid neoplasms and acute leukemia: Rationale and important changes. Blood 114: 937-951, 2009
3. Castaigne S, Chomienne C, Daniel MT, Ballerini P, Berger R, Fenaux P and Degos L: All-trans retinoic acid as a differentiation therapy for acute promyelocytic leukemia. I. Clinical results. Blood 76: 1704-1709, 1990.

4. Chen Z, Tong JH, Dong S, Zhu J, Wang ZY and Chen SJ: Retinoic acid regulatory pathways, chromosomal translocations and acute promyelocytic leukemia. Genes Chromosomes Cancer 15: $147-156,1996$

5. Jones ME and Saleem A: Acute promyelocytic leukemia. A review of literature. Am J Med 65: 673-677, 1978.

6. Cunningham I, Gee TS, Reich LM, Kempin SJ, Naval AN and Clarkson BD: Acute promyelocytic leukemia: Treatment results during a decade at Memorial Hospital. Blood 73: 1116-1122, 1989.

7. Sanz MA, Jarque I, Martin G, Lorenzo I, Martínez J, Rafecas J, Pastor E, Sayas MJ, Sanz G and Gomis F: Acute promyelocytic leukemia. Therapy results and prognostic factors. Cancer 61: 7-13, 1988.

8. Fenaux P, Wang ZZ and Degos L: Treatment of acute promyelocytic leukemia by retinoids. Curr Top Microbiol Immunol 313: 101-128, 2007.

9. Kamimura T, Miyamoto T, Harada M and Akashi K: Advances in therapies for acute promyelocytic leukemia. Cancer Sci 102: 1929-1937, 2011.

10. Looi CY, Arya A, Cheah FK, Muharram B, Leong KH, Mohamad K, Wong WF, Rai N and Mustafa MR: Induction of apoptosis in human breast cancer cells via caspase pathway byvernodalol isolated from Centratherum anthelminticum (L.) seeds. PLoS One 8: e56643, 2013.

11. Ananda Sadagopan SK, Mohebali N, Looi CY, Hasanpourghadi M, Pandurangan AK, Arya A, Karimian H and Mustafa MR: Forkhead box transcription factor (FOXO3a) mediates the cytotoxic effect ofvernodalol in vitro and inhibits the breast tumor growth in vivo. J Exp Clin Cancer Res 34: 147, 2015.

12. Ashok P, Koti BC, Thippeswamy AH, Tikare VP, Dabadi P and Viswanathaswamy AH: Evaluation of antiinflammatory activity of Centratherum anthelminticum (L) Kuntze seed. Indian J Pharm Sci 72: 697-703, 2010.

13. Purnima A, Koti BC, Tikare VP, Viswanathaswamy AH, Thippeswamy AH and Dabadi P: Evaluation of analgesic and antipyretic activities of Centratherum anthelminticum (L) Kuntze seed. Indian J Pharm Sci 71: 461-464, 2009.

14. Sharma S and Mehta BK: In vitro antimicrobial efficacy of Centratherum anthelminticum seeds extracts. J Hyg Epidemiol Microbiol Immunol 35: 157-161, 1991.

15. Singhal KC, Sharma S and Mehta BK: Antifilarial activity of Centratherum anthelminticum seed extracts on Setaria cervi. Indian J Exp Biol 30: 546-548, 1992.

16. Arya A, Achoui M, Cheah SC, Abdelwahab SI, Narrima P, Mohan S, Mustafa MR and Mohd MA: Chloroform fraction of Centratherum anthelminticum (L.) seed inhibits tumor necrosis factor alpha and exhibits pleotropic bioactivities: Inhibitory role in human tumor cells. Evid Based Complement Alternat Med 2012: 627256, 2012.

17. Manning BD and Cantley LC: AKT/PKB signaling: Navigating downstream. Cell 129: 1261-1274, 2007.

18. Petrie K, Zelent A and Waxman S: Differentiation therapy of acute myeloid leukemia: Past, present and future. Curr Opin Hematol 16: 84-91, 2009.

19. Rabe T, Mullholland D and van Staden J: Isolation and identification of antibacterial compounds from Vernonia colorata leaves. J Ethnopharmacol 80: 91-94, 2002.

20. Looi CY, Moharram B, Paydar M, Wong YL, Leong KH, Mohamad K, Arya A, Wong WF and Mustafa MR: Induction of apoptosis in melanoma A375 cells by a chloroform fraction of Centratherum anthelminticum (L.) seeds involves NF-kappaB, p53 and Bcl-2-controlled mitochondrial signaling pathways. BMC Complement Altern Med 13: 166, 2013.

21. Kasim LS, Ferro V, Odukoya OA, Ukpo GE, Seidel V, Gray AI and Waigh R: Cytotoxicity of isolated compounds from the extracts of Struchium sparganophora (Linn) Ktze asteraceae. Pak J Pharm Sci 24: 475-478, 2011.

22. Kupchan SM, Hemingway RJ, Karim A and Werner D: Tumor inhibitors. XLVII. Vernodalol and vernomygdin, two new cytotoxic sesquiterpene lactones from Vernonia amygdalina Del. J Org Chem 34: 3908-3911, 1969.

23. Drexler HG: Review of alterations of the cyclin-dependent kinase inhibitor INK4 family genes p15, p16, p18 and p19 in human leukemia-lymphoma cells. Leukemia 12: 845-859, 1998. 
24. Pan MH, Lin CL, Tsai JH, Ho CT and Chen WJ: 3,5,3',4', 5'-pentamethoxystilbene (MR-5), a synthetically methoxylated analogue of resveratrol, inhibits growth and induces G1 cell cycle arrest of human breast carcinoma MCF-7 cells. J Agric Food Chem 58: 226-234, 2010

25. Weir NM, Selvendiran K, Kutala VK, Tong L, Vishwanath S, Rajaram M, Tridandapani S, Anant S and Kuppusamy P: Curcumin induces $\mathrm{G} 2 / \mathrm{M}$ arrest and apoptosis in cisplatin-resistant human ovarian cancer cells by modulating Akt and p38 MAPK. Cancer Biol Ther 6: 178-184, 2007.

26. Abbas T and Dutta A: p21 in cancer: Intricate networks and multiple activities. Nat Rev Cancer 9: 400-414, 2009.

27. Furnari B, Blasina A, Boddy MN, McGowan CH and Russell P: $\mathrm{Cdc} 25$ inhibited in vivo and in vitro by checkpoint kinases Cds1 and Chk1. Mol Biol Cell 10: 833-845, 1999.

28. Innocente SA, Abrahamson JL, Cogswell JP and Lee JM: p53 regulates a G2 checkpoint through cyclin B1. Proc Natl Acad Sci USA 96: 2147-2152, 1999

29. McConkey DJ and Orrenius S. Signal transduction pathways in apoptosis. Stem Cells 14: 619-311, 1996.

30. Yang J, Liu X, Bhalla K, Kim CN, Ibrado AM, Cai J, Peng TI, Jones DP and Wang X: Prevention of apoptosis by Bcl-2: Release of cytochrome c from mitochondria blocked. Science 275: 1129-1132, 1997.

31. Martini M, De Santis MC, Braccini L, Gulluni F and Hirsch E: PI3K/AKT signaling pathway and cancer: An updated review. Ann Med 46: 372-383, 2014.

32. Vivanco I and Sawyers CL: The phosphatidylinositol 3-Kinase AKT pathway in human cancer. Nat Rev Cancer 2: 489-501, 2002.

33. Han S, Zhang G, Li M, Chen D, Wang Y, Ye W and Ji Z: L-securinine induces apoptosis in the human promyelocytic leukemia cell line HL-60 and influences the expression of genes involved in the PI3K/AKT/mTOR signaling pathway. Oncol Rep 31: 2245-2251, 2014.
34. Hildebrandt MA, Yang H, Hung MC, Izzo JG, Huang M, Lin J, Ajani JA and Wu X: Genetic variations in the PI3K/PTEN/ $\mathrm{AKT} / \mathrm{mTOR}$ pathway are associated with clinical outcomes in esophageal cancer patients treated with chemoradiotherapy. J Clin Oncol 27: 857-871, 2009.

35. Ma W, Wang DD, Li L, Feng YK, Gu HM, Zhu GM, Piao JH, Yang Y, Gao X and Zhang PX: Caveolin-1 plays a key role in the oleanolic acid-induced apoptosis of HL-60 cells. Oncol Rep 32: 293-301, 2014.

36. Burgering BM and Coffer PJ: Protein kinase B (c-Akt) in phosphatidylinositol-3-OH kinase signal transduction. Nature 376: 599-602, 1995.

37. Wick MJ, Dong LQ, Riojas RA, Ramos FJ and Liu F: Mechanism of phosphorylation of protein kinase B/Akt by a constitutively active 3-phosphoinositide-dependent protein kinase-1. J Biol Chem 275: 40400-40406, 2000.

38. Aoki M, Blazek E and Vogt PK: A role of the kinase mTOR in cellular transformation induced by the oncoproteins $\mathrm{P} 3 \mathrm{k}$ and Akt. Proc Natl Acad Sci USA 98: 136-141, 2001.

39. Ali IU, Schriml LM and Dean M: Mutational spectra of PTEN/MMAC1 gene: A tumor suppressor with lipid phosphatase activity. J Natl Cancer Inst 91: 1922-1932, 1999.

40. Li J, Yen C, Liaw D, Podsypanina K, Bose S, Wang SI, Puc J, Miliaresis C, Rodgers L, McCombie R, et al: PTEN, a putative protein tyrosine phosphatase gene mutated in human brain, breast and prostate cancer. Science 275: 1943-1947, 1997.

(i) (9) This work is licensed under a Creative Commons

EY NO NO Attribution-NonCommercial-NoDerivatives 4.0 International (CC BY-NC-ND 4.0) License. 